\title{
Visible light emission from semiconducting polymer diodes
}

\author{
D. Braun and A. J. Heeger \\ Institute for Polymers and Organic Solids, University of California at Santa Barbara, \\ Santa Barbara, California 93106
}

\begin{abstract}
We report visible light emission from Shottky diodes made from semiconducting polymers, confirming the discovery by the Cambridge group [Nature 347, 539 (1990)]. Our results demonstrate that light-emitting diodes can be fabricated by casting the polymer film from solution with no subsequent processing or heat treatment required. Electrical characterization reveals diode behavior with rectification ratios greater than $10^{4}$. We propose that tunneling of electrons from the recitifying metal contact into the gap states of the positive polaron majority carriers dominates current flow and provides the mechanism for light emission.
\end{abstract}

We confirm the results of Burroughes et al. ${ }^{1}$ and demonstrate that electroluminescence from conjugated polymer diodes is a more general phenomenon. We report light emission from diodes fabricated with MEH-PPV, poly (2-methoxy, 5-(2'-ethyl-hexoxy)-1, 4-phenylenevinylene) ${ }^{2}$ MEH-PPV offers the advantage of being soluble in the conjugated form in organic solvents. We describe device fabrication, simplified because of direct casting of the semiconducting polymer from solution, and we present the results of electrical and optical characterization of the devices, including the temperature dependence of the electroluminescence (EL) and photoluminescence (PL) spectra.

The light-emitting diodes (LEDs) consist of a rectifying metal contact on the front surface of an MEH-PPV film on a glass substrate, partially coated with a layer of indium/tin-oxide (ITO), the "ohmic" contact. The MEHPPV films are prepared by spin casting from tetrahydrofuran or xylenes solutions containing 1\% MEH-PPV by weight. The resulting MEH-PPV films have uniform surfaces with thicknesses near $1200 \AA$. Metal contacts (indium or calcium) are deposited on top of the polymer films by vacuum evaporation at pressures below $4 \times 10^{-7}$ Torr yielding active areas of $0.04 \mathrm{~cm}^{2}$. All processing steps are carried out in a nitrogen atmosphere. Silver paint or indium solder is used to connect wires to the electrodes. Spectroscopic measurements use a single-grating monochromator followed by a photomultiplier tube (PMT) as detector. The modulated signal from the PMT is processed by a lock-in amplifier tuned to the modulation frequency. For EL spectra, modulation is achieved by applying a sinusoidal voltage superposed on a dc voltage. For PL spectra, the polymer is pumped by an argon ion laser (457.9 nm); PL modulation is achieved by mechanically chopping the pump beam. The measurements are carried out with the LEDs in a vacuum cryostat at pressures below $10^{-4}$ Torr.

Figure 1 shows the EL spectra obtained with $3 \mathrm{~V} \mathrm{AC}$ superposed (at $681 \mathrm{~Hz}$ ) on $13 \mathrm{~V}$ forward bias. The roomtemperature EL peaks near $2.1 \mathrm{eV}$ with a hint of a second peak above $1.9 \mathrm{eV}$. At $90 \mathrm{~K}$, the intensity increases and shifts to the red, and the two peaks become clearly resolved. Figure 1 also compares the EL and PL spectra at low temperature. The two spectra are essentially identical.
Current versus voltage $(I-V)$ characteristics are shown for the polymer/In diode in the inset to Fig. 2. While ramping the applied bias, yellow-orange light becomes visible to the eye just below $9 \mathrm{~V}$ forward bias (no light is observed under reversed bias). Above $15 \mathrm{~V}$, the rectification ratio exceeds $10^{4}$. Independent evidence from the temperature dependence of the $I-V$ characteristics suggests that in diodes fabricated from semiconducting polymers, carrier injection takes place via tunneling. ${ }^{3}$ Standard tunneling theory predicts ${ }^{4}$

$$
I \propto V^{2} \exp \left(-b / Y^{\gamma}\right),
$$

where $b$ is a fitting parameter that depends on the shape and height of the tunneling barrier. Thermionic emission theory predicts ${ }^{4}$

$$
I \propto \exp (-e V / n K T),
$$

where $e$ is the electron charge, $K$ is the Boltzmann constant, and $n$ is the quality factor. Figure 2 displays the data, plotted as $\ln \left(I / V^{2}\right)$ vs $1 / V$; the linear behavior is consistent with the tunneling model of Eq. (1). The temperature dependence of the EL shown in Fig. 1 is consistent with the

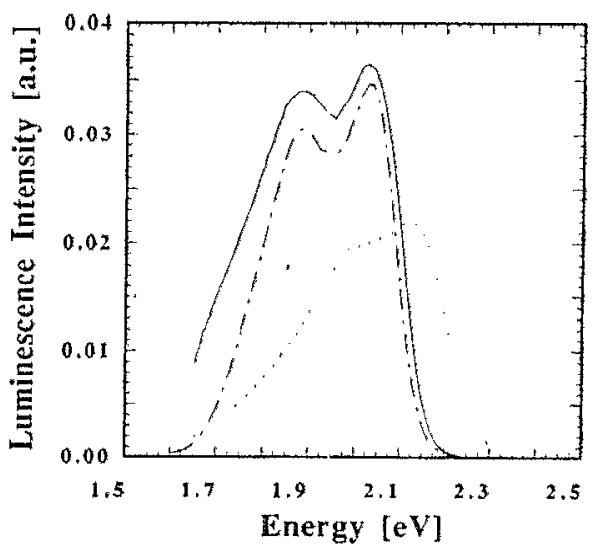

FIG. 1. Electroluminescence intensity vs photon energy at $300 \mathrm{~K}$ (dotted) and $90 \mathrm{~K}$ (solid) for irdium/MEH-PPV diode under a forward bias of $13 \mathrm{~V}$ dc with $3 \mathrm{~V}$ ac superposed at $681 \mathrm{~Hz}$; photoluminescence spectrum (dashed) at $90 \mathrm{~K}$ shown for comparison. 


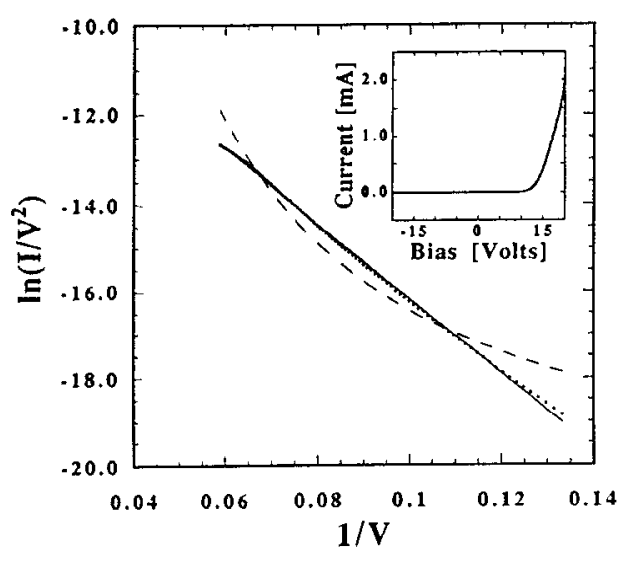

FIG. $2 . \ln (I / V)$ vs $1 / V$ for the indium/MEH-PPV diode; the solid curve is the best fit to the tunneling expression [Eq. (1)]; the dashed curve is the best fit to the expression for thermionic emission [Eq. (2)]. The inset shows the current vs voltage characteristic over the full voltage range.

tunneling model; an increase in luminescence efficiency at higher temperatures would be expected with a thermally activated process.

Extensive studies of the spectral features and the anisotropy of the PL from spin-cast films of MEH-PPV and from MEH-PPV oriented by mesoepitaxy in blends with polyethylene reveal that the PL observed in oriented MEH-PPV is highly polarized parallel to the chain orientation axis ( $>60: 1$ ratio), so the luminescence center is an intra-chain exciton (alternately described as a neutral bipolaron) formed from a positive and a negative polaron. ${ }^{5}$ Since the spectra obtained from PL and EL are identical, the luminescent center is the same in the two cases. The optical properties of conjugated polymers have been alternatively described in terms of an interband transition (followed by structural relaxation $)^{6}$ or in terms of a correlated exciton model. ${ }^{7}$ Since the line shape of the absorption spectrum of oriented MEH-PPV in polyethylene is accurately described by that predicted for the $\pi-\pi^{*}$ transition in a quasi-one-dimensional semiconductor, ${ }^{5}$ we approach the mechanism of light emission from the band model.

In the LED configuration, carrier injection at the rectifying contact can lead to neutral (bipolaron) excitons in two ways. Thermal excitation of electrons from the metal enables injection into the conduction band of the polymer (mechanism 2 in Fig. 3). After thermionic emission, selflocalization will lead to the formation of a negative polaron which recombines with a positive polaron to form the excited state of the neutral bipolaron exciton; a state with lower total energy than the two oppositely charged polarons because of the Coulomb attraction. The excited state decays radiatively as sketched in inset A of Fig. 3. Alternatively, when positive polaron majority carriers diffuse to the depletion layer, electrons from the rectifying contact can tunnel through the barrier directly into the polaron gap states. Tunneling into the upper gap state (mechanism 1 in Fig. 3) is followed by structural relaxation to the excited state of the neutral bipolaron with radiative decay (inset A of Fig. 3). Tunneling into the lower gap state results in an unstable configuration (inset $\mathrm{B}$ ); nonradiative
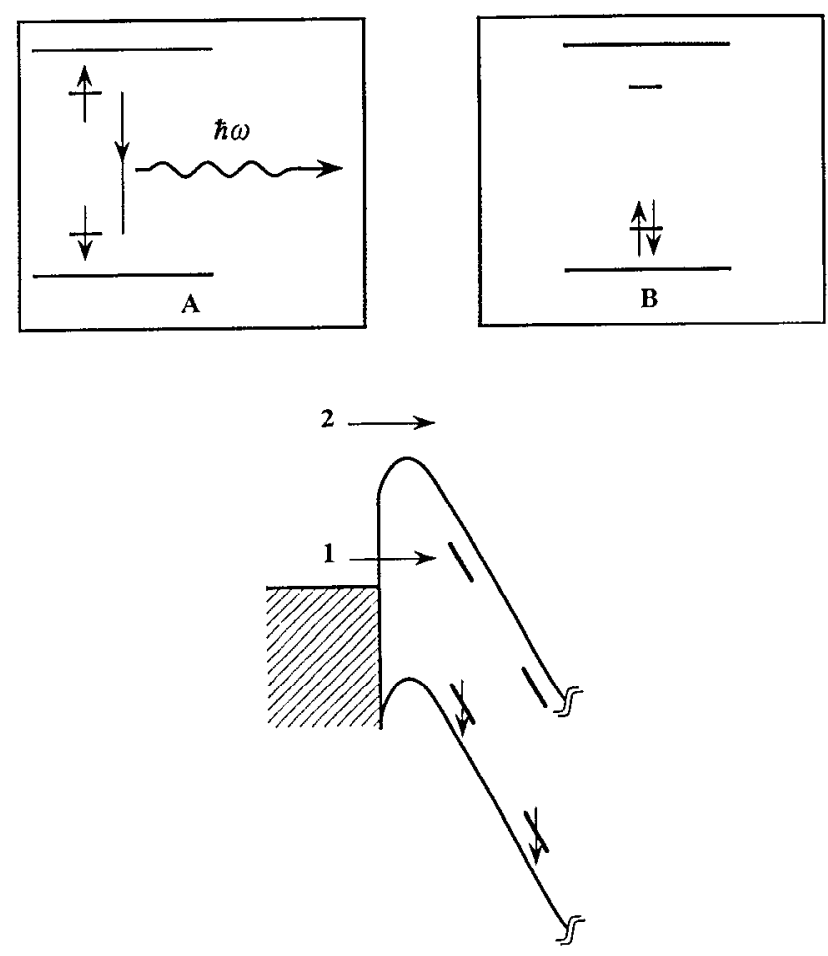

FIG. 3. Channel for lumincsecnec and possible current fiow mechanisms: the band diagram illustrates electron injection either by thermionic emission (2) into conduction band and subsequent relaxation or by direct tunneling (1) into upper polaron level. Inset A shows decay of the excited state of the neutral bipolaron through radiative recombination, and inset $B$ shows the final state of the neutral bipolaron which decays nonradiatively.

structural relaxation causes the gap states to merge into the valence and conduction bands. We propose, therefore, that the tunneling-injection mechanism depicted in Fig. 3 dominates in the conducting polymer LEDs.

Based on the diagrams sketched in Fig. 3, the modest EL efficiency results from the competition between the radiative process (tunneling into the upper gap state) and the nonradiative process (tunneling into the lower gap state). Since the chemical potential in the $p$-type polymer is set by the partial occupancy of the lower polaron level, this asymmetry may be an intrinsic feature of the metalpolymer diodes. This scheme suggests that increasing the barrier height for majority carriers and decreasing the barrier height for electrons at the rectifying metal contact would favor the light emission channel.

The data of Fig. 4 verify this prediction. Figure 4 displays the EL intensity as a function of current flow under increasing forward bias. The EL intensity emitted by LEDs with calcium electrodes (work function $=3 \mathrm{eV}^{4}$ ) as the rectifying contact exceeds by almost an order of magnitude the EL intensity emitted by LEDs with indium electrodes ( work function $=4.2 \mathrm{eV}^{4}$ ). The quantum efficiency (as determined with a calibrated silicon photodiode and corrected for the spectral response and the solid angle of the collecting optics) at $1 \mathrm{~mA}$ is $\approx 5 \times 10^{-4}$ photons per electron for indium electrodes and $\approx 0.01$ photons per electron for calcium electrodes. The emission from the calcium/ 


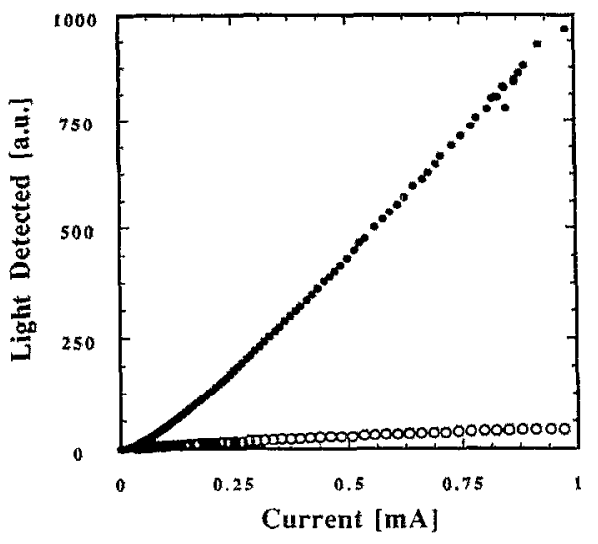

FIG. 4. Emitted light intensity recorded by calibrated Si photodiode vs current flowing through polymer LEDs with calcium electrodes (dots) and indium electrodes (circles).

MEH-PPV LEDs is easily seen in a lighted room at $4 \mathrm{~V}$ forward bias.

The recent invention ${ }^{1}$ of conducting polymer LEDs expands the possible applications for conducting polymers into the area of active light sources. Controlling the energy gap of the polymer, either through the judicious choice of the conjugated backbone structure or through side-chain functionalization, should make possible a variety of colors. Moreover, because of processing advantages of semiconductors cast from solution, large active areas can be envisioned. Thus, LEDs labricated from conducting polymers offer a number of potential advantages to future technology.

We thank F. Wudl and Z. Ni for preparing the MEHPPV and H. Kroemer, T. Hagler, K. Pakbaz, L. Smilowitz, and $\mathrm{K}$. Voss for yaluable discussions and assistance. The research was funded by the Office of Naval Research (N00014-83-K-0450). Device fabrication utilized facilities obtained through an equipment grant from Showa Denko K.K.

'J. H. Burroughes, D. D. C. Bradley, A. R. Brown, R. N. Marks, K. Mackay, R. H. Friend, P. L. Bums, and A. B. Holmes, Nature 347, 539 (1990).

${ }^{2}$ F. Wudl, P..M. Allemand, G. Srdanov, Z. Ni, and D. McBranch, Materials for Non-linear Optics.Chemical Perspectives (1991) (unpublished).

${ }^{3}$ H. Tomozawa, D. Braun, S. D. Phillips, R. Worland, A. J. Heeger, and H. Kroemer, Synth. Met. 28, C687 (1989).

${ }^{4}$ S. M. Sze, Physics of Semiconductor Devices (Wiley, New York, 1981).

${ }^{5}$ T. W. Hagler, K. Pakbax, J. Moulton, F. Wudl, P. Smith, and A. J. Heeger, Polym. Commun, (1991) (unpublished).

${ }^{6}$ A. J. Heeger, S. Kivelson, J. R. Schrieffer, and W.-P. Su, Rev. Mod. Phys. 60, 781 (1988).

${ }^{7}$ U. Rauscher, II. Bässler, D. D. C. Bradley, and M. Hennecke, Phys. Rev. B 42, 9830 (1990). 